\title{
Roll-your-own smoke yields: theoretical and practical aspects
}

\author{
Keith G Darrall, John A Figgins
}

\begin{abstract}
Objective-To identify the key parameters that influence smoke yields from rollyour-own (RYO) cigarettes and to compare smoke yields of cigarettes made under laboratory conditions with those made by habitual RYO consumers.

Design and setting-One-way parametric variations in the laboratory-based production of RYO cigarettes complemented by a consumer survey conducted in a busy street at Romford, Essex, United Kingdom.

Subjects-26 habitual RYO consumers.

Main outcome measures-Cigarette weights, puff numbers, and yields (carbon monoxide, nicotine, and tar).

Results-Smoke yields vary for specimen changes in weight of tobacco used, paper porosity, and the incorporation of a filter in the cigarette. Yields of cigarettes produced by 26 RYO smokers ranged from 9.9 to $21.0 \mathrm{mg}$ tar per cigarette and from 0.9 to $1.8 \mathrm{mg}$ nicotine per cigarette, and were generally lower than yields of laboratory-produced RYO cigarettes. Conclusions-Laboratory studies can provide useful information concerning the parameters that affect smoke yields of RYO cigarettes such as the incorporation of a filter to reduce yields. However, such studies must be complemented by surveys of cigarettes made by actual current RYO smokers. In one such investigation, it was found that the mean tar yields from cigarettes produced by $57 \%$ of the smokers were above the current maximum of $15 \mathrm{mg}$ per cigarette for manufactured cigarettes. Currently $8 \%$ of manufactured cigarettes in the UK have a declared nicotine yield of greater than $1.1 \mathrm{mg}$ per cigarette whereas $77 \%$ of RYO smokers produced cigarettes with a nicotine yield greater than this value.

(Tobacco Control 1998;7:168-175)
\end{abstract}

Keywords: roll-your-own cigarettes, smoke yield, carbon monoxide, tar, nicotine

Laboratory of the Government Chemist, Teddington, UK K G Darrall J A Figgins

Correspondence to: K G Darrall, Laboratory of the Government Chemist

(LGC), Queens Road, Teddington, Middlesex TW11 0LY, UK. kgd@lgc.co.uk recorded as 3050 tonnes in $1994{ }^{1}$ However it is also known that a high level of smuggling now occurs, ${ }^{12}$ which suggests that published sales volumes will tend to underestimate actual consumption. The number of hand-rolled cigarettes made annually in the United Kingdom can be roughly estimated assuming an average weight of tobacco used per cigarette as $490 \mathrm{mg}^{3}$. It may also be assumed that tobacco smoked in this form has toxic properties similar to those of tobacco smoked in manufactured cigarettes. Due to lack of analytical information, it has not yet been possible to inform the smoking public in any detail about the hazards associated with either particular brands of tobacco and smoking accessories, or particular rolling techniques and habits. It has been reported ${ }^{4}$ that in the United Kingdom in 1994 more than $20 \%$ of male smokers used roll-your-own products compared with less than $4 \%$ of female smokers. Such smokers tend to be concentrated in the lower social classes as measured by the deprivation index. ${ }^{4}$

The health hazards associated with cigarette smoking have been widely documented, particularly the link to lung cancer, ${ }^{5}$ and also a wide range of other adverse effects ${ }^{67}$ grouped as smoking-related diseases. A study in Norway ${ }^{8}$ has reported an increased risk of lung cancer in hand-rolled tobacco smokers compared with those smoking manufactured cigarettes. In addition to lung cancer, there have been reports ${ }^{9-12}$ of other specific cancers linked to use of hand-rolled tobacco. After adjustment for alcohol consumption, which is a confounding cause, an association has been noted between the use of hand-rolling tobacco and the risk of oesophageal cancer. This is, however, more prevalent in hand-rollers of dark air-cured tobaccos, but nevertheless a smaller but measurable increased risk was identified in hand-rollers using blond or Virginia tobacco. ${ }^{9}{ }^{10}$ It has also been reported ${ }^{11}$ that hand-rolled cigarette smoking is associated with increased risk of cancer of the mouth, pharynx, and larynx. A report ${ }^{12}$ from Australia concludes that although at present there is insufficient epidemiological evidence to link large-bowel cancer with smoking, nevertheless an excess risk was observed for male smokers of hand-rolled cigarettes and for cigar and pipe smokers. We would suggest that the observations in references 9-12 are likely to be caused by a combination of ingestion of tobacco particles and smoke inhalation.

During the past two decades, strenuous measures have been taken to reduce the mainstream smoke yields, and in particular the tar yield, of manufactured cigarettes. ${ }^{13}$ This has been translated into European legislation by 
enforcing a maximum tar yield for cigarettes which can be sold within the European Community. A $15 \mathrm{mg}$ per cigarette maximum tar yield was introduced in 1991 and a further reduction to $12 \mathrm{mg}$ per cigarette was scheduled to come into force on 1 January 1998.

Unlike manufactured cigarettes, there are at present no internationally recognised methods for the determination of the smoke yields of hand-rolled cigarettes. The many physical parameters of a manufactured cigarette such as weight of tobacco, diameter, tobacco packing density, and porosity of wrapping paper are closely controlled, resulting in a reasonably reproducible product which in turn gives defined yields when smoked using standard smoking machine methodology. However, the production of the hand-rolled cigarette is largely under the control of the person making it. There is, therefore, the opportunity for wide differences in the parameters of the finished articles, which is likely to result in a wide range of smoke yields as measured under laboratory conditions.

Few published studies have so far addressed the issue of smoke yields from hand-rolling tobaccos. In Europe some data have been produced in the form of in-house laboratory reports. ${ }^{14}{ }^{15}$ Examples of other scattered reports of hand-rolled cigarette yields include those from Canada ${ }^{16}$ and Thailand. ${ }^{17}$ However, the practices and products employed in these countries are not comparable to those in the United Kingdom, and therefore the reports are of limited applicability here. A detailed study of roll-your-own practices in the Netherlands has been published recently ${ }^{18}$ but again, such continental practices do not reflect United Kingdom behaviour where it is customary to use much lower unit weight of tobacco for each cigarette.

It is useful to describe those who smoke cigarettes that they have themselves made from hand-rolling tobacco as roll-your-own (RYO) smokers, a category that includes those who use a rolling machine. The first phase of this project, in conjunction with strictly defined tobacco-rolling procedures, examined selected RYO cigarette parameters that are known to influence smoke yields; the objective was to contribute to an understanding of how RYO smoke yields are influenced and of possible legislative implications. The smoking articles tested in this phase were produced with a commercially available rolling machine, designed for use with hand-rolling tobacco. The machine rolling procedure was adopted, where possible, to minimise unplanned variations in the cigarette making process, so as to allow the outcomes of planned variations in parameters to be observed more clearly.

The second phase of the project was complementary, in that smoke yields from cigarettes produced by members of the RYO smoking public were determined. Sufficient data representative of habitual use of hand-rolling tobaccos were produced to allow some tentative comparisons to be drawn with typical smoke yields from branded manufactured cigarettes. The analytical work was carried out between August 1993 and September 1995.

Standard smoking machine parameters were used for the determination of cigarette smoke yields. While yields obtained with these parameters are unlikely to represent those obtained from most individual human smoking patterns, it has been shown ${ }^{19}$ in work with manufactured cigarettes that the rank positions of cigarette brands with different tar yields do not change significantly when alternative smoking parameters are used. There seems little reason to doubt that this rank order will not extend from manufactured to RYO cigarettes, and therefore a comparison using the standard smoking parameters can be justified.

\section{Methods}

APPARATUS

Tobaccos, papers and filters were purchased from a local specialist retail outlet. The brand of hand-rolling tobacco used in this study was one of the market leaders in the United Kingdom at the inception of the study. The papers and filters used were brands that are widely available within the United Kingdom.

A fully harmonised Filtrona F350 smoking machine (Filtrona Instruments and Automation Ltd, Milton Keynes, Bucks) was used to smoke cigarettes. This machine has 20 smoking channels in a linear array and was equipped with a non-dispersive infra-red carbon monoxide analyser. The smoking machine was calibrated to take a $35 \mathrm{~cm}^{3}$ puff of two seconds' duration at intervals of 60 seconds, and thus conformed with the international parameters for machine smoking. ${ }^{20}$ It was operated in a controlled environment of relative humidity $60 \pm 5 \%$ and a temperature of $22 \pm 2^{\circ} \mathrm{C}$.

Water and nicotine were determined using a Varian Model 3400 gas chromatograph equipped with a model 8100 autosampler (Varian Ltd, Walton on Thames, Surrey). Each sample aliquot injected was split at a glass-lined $\mathrm{T}$ piece onto appropriate columns. The water determination used a $2.0 \mathrm{~m} \times 2 \mathrm{~mm}$ id nickel column, packed with Porapak QS, and connected to a thermal conductivity detector. The nicotine determination used a nickel column of similar dimensions, packed with $10 \%$ Carbowax $20 \mathrm{M}$ plus $2 \%$ potassium hydroxide on Chromosorb WHP, and connected to a flame ionisation detector.

The tobacco conditioning environment was controlled with a Sanyo Gallenkamp Model FE300H cabinet; a temperature of $22 \pm 1^{\circ} \mathrm{C}$ was used with a relative humidity of either $60 \pm 2 \%$ or $75 \pm 3 \%$ (as specified below). Cigarette pressure drop measurements were made using a Filtrona model PDAVHP pressure drop meter. Paper porosity measurements were made by the Filtrona Instruments and Automation Laboratory, Jarrow, Tyne \& Wear.

The cigarette rolling machine used was the Automatic Cigarette Rolling Box manufactured by Rizla United Kingdom Ltd, Pontypridd, Mid-Glamorgan. 
DEFINED CIGARETTE-MAKING PROTOCOLS Cigarettes were made using a Rizla automatic cigarette maker. The required amount of tobacco ( $0.75 \mathrm{~g}$ unless otherwise stated) was placed evenly along the length of a paper inside the maker, the closing of which ejects the fully rolled cigarette. The finished cigarette was then weighed, using an analytical balance, to ascertain the actual weight of tobacco used, a correction being applied for the average weight of a paper. Cigarettes were discarded if the weight of tobacco differed by more than $0.015 \mathrm{~g}$ from the intended weight. The pressure drop across each cigarette was determined on completion of rolling each batch of cigarettes; this measurement indicates the packing density, and, in practical terms, cigarettes with unusually high or low pressure drops are likely to give correspondingly variable yields when machine smoked. Ten to $15 \%$ of the machine-rolled cigarettes were excluded because the corresponding pressure drops fell at the extremes of the range.

For additional experiments requiring cigarettes to be made with $0.50 \mathrm{~g}$ of tobacco, the making procedure was fully manual because it proved difficult to obtain reproducible machine-rolled cigarettes with the lower quantity of tobacco. While this method will reflect the technique of a single RYO user, it obviously cannot necessarily be assumed to represent the technique of other such rollers. A similar weight criterion was used as for the $0.75 \mathrm{~g}$ items above. As might be expected there was a relatively wide range of pressure drops for the hand-rolled cigarettes; only clear outliers were excluded to minimise distortion of the range of yields likely to be produced in practice.

Cigarettes were produced in batches large enough to allow four to six filter traps on the smoking machine to be used simultaneously. The cigarettes were then conditioned for 20 hours at $60 \pm 2 \%$ relative humidity and $22 \pm 1{ }^{\circ} \mathrm{C}$ before smoking.

SMOKING AND ANALYTICAL PROTOCOLS

Machine smoking was carried out according to the standard procedure, ${ }^{21}$ except that only four cigarettes were smoked through each $44 \mathrm{~mm}$ Cambridge filter trap. This modification was introduced after preliminary experiments indicated the possibility in some cases of the filter pad becoming overloaded with particulate matter. The standard method requires five cigarettes per determination. The cigarettes were smoked to the standard butt length for plain cigarettes of $23 \mathrm{~mm}$. Cigarettes incorporating a filter (length $15 \mathrm{~mm}$ ) were also smoked to the same butt length.

The total particulate matter (TPM) collected in each filter trap was weighed, then extracted with $20 \mathrm{ml}$ propan-2-ol. Nicotine and water in the extract were determined by standard gas chromatographic methods. ${ }^{22} 23$ Nicotine-free dry particulate matter (NFDPM, also known as "tar") was calculated, on a per cigarette basis, as:

TPM - [nicotine + water].
Carbon monoxide (CO) in the vapour phase of the smoke was determined by the standard non-dispersive infrared method. ${ }^{24}$ Nicotine was extracted from cigarette filter tips with methanol containing sodium hydroxide $(0.1 \% \mathrm{~m} / \mathrm{v})$, before being determined by gas chromatography.

SURVEY OF HAND-ROLLING TOBACCO USERS A market research organisation, the British Market Research Bureau (BMRB), was commissioned to organise the recruitment of RYO smokers who would roll specimen cigarettes, which could then be machinesmoked. From their database, BMRB identified an area (Romford, Essex) with an above average population of RYO smokers. On the basis of pre-existing market research data suggesting that there are substantially more male than female RYO users, ${ }^{4}$ BMRB concentrated their efforts towards the recruitment of male participants, and, in fact, only one participant was female. There were no age stipulations on people taking part.

During shopping hours on 9 September 1995, experienced RYO users were sought from a busy street and invited to roll 20 cigarettes each. Leading brands of both tobacco and paper were provided. It was impressed on each participant that the cigarettes should be as he or she would roll them for personal use. One participant normally used a rolling machine which he had with him, and also used it for this survey; all other participants rolled by hand. Cigarettes were produced without filters, although two participants said that they normally incorporated filters in their cigarettes. Twenty-five participants rolled 20 cigarettes as requested. One participant rolled 17 cigarettes.

At the time of producing each cigarette, the participants identified which end they considered to be the mouth end. Care was taken in subsequent handling to ensure that the integrity of this information was maintained and that finally the cigarettes would be smoked in the orientation intended.

The cigarettes made by each participant were individually weighed by laboratory staff on completion of each batch. The batches were then packed in separate airtight boxes for transportation back to the laboratory, and the gross weight of each box was recorded. On arrival at the laboratory, approximately two hours after the last cigarettes had been weighed on site, the gross weight of each box was again recorded. The cigarettes were stored at a relative humidity of $75 \pm 3 \%$ at $22 \pm 1^{\circ} \mathrm{C}$ until machine smoking. These conditions were used, on the basis of advice from the tobacco industry, to ensure that the cigarettes would remain at a constant moisture level over an extended period of time and have since been published as a Coresta standard procedure..$^{25}$

Machine smoking of the hand-rolled cigarettes was spread over three days (seven smoking runs). Cigarettes were drawn at random from each box of 20 to group them into five samples of four cigarettes for each participant. Each of the five samples from a 
box were smoked on a different smoking run, except that the contents of one box, the weight control, was left intact and then smoked with the other remaining samples on the final run. Four samples were smoked from the participant who only produced 17 cigarettes. Cigarettes were smoked on randomly determined channels of the smoking machine to the standard butt length of $23 \mathrm{~mm}$. Each smoking run included an in-house monitor cigarette on one channel as a quality control adjunct.

The hand-rolled cigarettes varied considerably in diameter. Each cigarette was matched individually to a labyrinth seal of the most appropriate diameter so as to minimise the possibility of leakage during smoking. In contrast to handmade cigarettes previously made in the laboratory, it was apparent that a significant number of the cigarettes in this study extinguished on occasion during the inter-puff intervals. These were re-lit at the appropriate time and, where necessary, allowance was made in carbon monoxide calculations for any puffs taken when a cigarette was not alight. In all other respects, the analytical procedures described for the initial study were followed.

\section{Results}

EXPERIMENTAL VALIDATION STUDIES

The scale of this project and the special properties of hand-rolling tobaccos necessitated control experiments to guard against long-term methodological drift and to determine appropriate tobacco storage conditions.

The nicotine content of the RYO tobacco brand, which was used extensively throughout the study, was determined in duplicate six times over the first 18 months of the study and again for the tobacco used for the public study. These results were used to indicate the extent to which longer term variations within the

Table 1 Variation of smoke yields (mg per cigarette) of machine-rolled cigarettes with conditioning interval at standard environmental conditions

\begin{tabular}{llll}
\hline Interval (hours) & CO & Nicotine & Tar \\
\hline$<8$ & $23.2(2.1)$ & $2.68(0.29)$ & $36.5(2.6)$ \\
20 & $24.0(1.1)$ & $2.98(0.11)$ & $36.1(1.5)$ \\
44 & $23.3(1.5)$ & $3.12(0.18)$ & $35.9(2.5)$ \\
\hline
\end{tabular}

All results are the mean yields for four determinations (sample standard deviations in parentheses). Cigarettes were conditioned for the tabulated intervals at $22^{\circ} \mathrm{C}$ and $60 \%$ relative humidity before machine smoking.

$\mathrm{CO}=$ carbon monoxide

Table 2 Association of smoke yields ( $m g$ per cigarette) with the weight of tobacco used and the choice of cigarette paper

\begin{tabular}{lllllll}
\hline $\begin{array}{l}\text { Tobacco } \\
\text { weight }(g)\end{array}$ & $\begin{array}{l}\text { Brand of } \\
\text { paper }\end{array}$ & $\begin{array}{l}\text { No of } \\
\text { results }\end{array}$ & CO & Nicotine & Tar & $\begin{array}{l}\text { Porosity } \\
\text { (Coresta } \\
\text { units) }\end{array}$ \\
\hline 0.75 & $\mathrm{~A}$ & 15 & $23.9(1.2)$ & $2.80(0.20)$ & $35.7(2.2)$ & 20 \\
0.75 & $\mathrm{~B}$ & 15 & $22.2(2.4)$ & $2.68(0.11)$ & $31.4(1.5)$ & 19 \\
0.75 & $\mathrm{C}$ & 10 & $18.5(1.8)$ & $2.26(0.12)$ & $26.6(1.9)$ & 77 \\
0.5 & $\mathrm{~A}$ & 10 & $18.5(1.8)$ & $2.00(0.13)$ & $23.8(1.7)$ & 20 \\
0.5 & $\mathrm{~B}$ & 10 & $14.6(2.0)$ & $2.10(0.19)$ & $23.5(3.0)$ & 19 \\
0.5 & $\mathrm{C}$ & 10 & $14.8(1.9)$ & $1.85(0.10)$ & $20.7(1.7)$ & 77 \\
\hline
\end{tabular}

All yield figures are means from the stated number of replicates (standard deviations in parentheses). Porosity values are the means of 20 measurements for each brand of paper.

${ }^{\star}$ Ratio of the air flow (volume per unit time) per unit of surface area of the cigarette paper to the difference in pressure across the paper. This is expressed in cubic centimetres per minute per square centimetre per kilopascal (Coresta units).

$\mathrm{CO}=$ carbon monoxide brand might affect the results of analyses carried out in a relatively short interval. Samples of each batch purchased and used in the study were examined. The mean of the seven determinations made was $24.4 \mathrm{mg}$ nicotine per $\mathrm{g}$ of tobacco as packed and the coefficient of variation was $2.9 \%$. We concluded that there was no significant between-batch variation in the nicotine content of the tobacco over the period of the study.

Hand-rolling tobacco products are packed at a relatively high moisture content (generally about $20 \%$, compared with $13 \%$ for manufactured cigarettes). Changes in the moisture content of the tobacco will affect the rate of burn during machine smoking and will contribute unnecessary variations to the determined smoke yields. Because it was not convenient to perform smoking experiments together with the necessary analytical determinations immediately after preparation of the RYO articles, it was necessary to select a conditioning environment as part of the standardised cigarettemaking protocol (see Methods). A check was made on the effects of conditioning RYO smoking articles for various intervals at the standard relative humidity and temperature used for manufactured cigarettes. Tar and CO yields were found to be little affected by the choice of conditioning interval, whereas noticeable nicotine yield increases were found with conditioning interval (table 1 ). The shortest practical interval, 20 hours, was used as part of the standardised protocol. It must be concluded that this is likely to have led to some overestimation of nicotine yield in this part of the study.

ASSOCIATION BETWEEN PAPER TYPE AND SMOKE YIELDS

Smoke yields were measured on cigarettes machine-rolled in the laboratory, each containing $0.75 \mathrm{~g}$ of tobacco, for three papers: one paper (B) was a market-leading brand, the second (A) was from a different manufacturer but of similar porosity, while the third (C) was of relatively high porosity (table 2 ). Smoke yields for the two papers of similar porosity were themselves similar, particularly $\mathrm{CO}$ and nicotine yields. In line with theoretical predictions for the paper of high porosity, the smoke yields were markedly lower.

Similar experiments were carried out with $0.5 \mathrm{~g}$ of tobacco per cigarette. Smoke yields were less clearly dependent on porosity when $0.5 \mathrm{~g}$ of tobacco per cigarette was used instead of $0.75 \mathrm{~g}$; nevertheless, the overall trends were similar (table 2).

\section{EFFECT OF INCORPORATING A FILTER}

Cigarettes were machine-rolled under laboratory conditions with the same leading brand of tobacco and paper (B), and two studies on the effects of incorporating a filter in the smoking article were undertaken. In the first, each cigarette contained $0.75 \mathrm{~g}$ of tobacco, and smoke yields with or without inclusion of a standard diameter filter were compared. The filter had little effect on CO yield, but reduced nicotine 
Table 3 The relationship between smoke yields ( $m g$ per cigarette) and the use of filters under standardised cigarette-making conditions

\begin{tabular}{lllllll}
\hline $\begin{array}{l}\text { Tobacco } \\
\text { weight }(g)\end{array}$ & Filter used & $\begin{array}{l}\text { No of } \\
\text { results }\end{array}$ & CO & Nicotine & Tar & $\begin{array}{l}\text { Nicotine } \\
\text { retained (\%) }\end{array}$ \\
\hline 0.75 & None & 15 & $23.9(1.2)$ & $2.80(0.20)$ & $35.7(2.2)$ & NA \\
0.75 & Standard & 15 & $24.1(2.1)$ & $1.46(0.17)$ & $19.3(2.0)$ & ND \\
0.59 & Standard & 12 & $21.1(1.4)$ & $1.22(0.09)$ & $16.0(1.5)$ & $57(6)$ \\
0.59 & Slimline & 12 & $24.4(0.5)$ & $2.49(0.21)$ & $28.8(0.7)$ & $34(1)$ \\
\hline
\end{tabular}

The smoke yield figures are means from the stated number of replicates (standard deviations in parentheses). Nicotine retained on the filter is expressed as a percentage of the sum of smoke nicotine and nicotine retained on the filter; these figures are each the mean (standard deviation in parentheses) of three determinations.

$\mathrm{CO}=$ carbon monoxide; $\mathrm{NA}=$ not applicable $\mathrm{ND}=$ not determined.

Table 4 Association between the weight of hand-rolled cigarettes and storage time at $75 \%$ relative humidity and $22^{\circ} \mathrm{C}$

\begin{tabular}{lll}
\hline Storage time (days) & Mean weight of 15 boxes $(g)$ & Weight of box 26 $(g)$ \\
\hline 0 (on site) & 73.15 & 72.43 \\
0 (laboratory) & 73.14 & 72.41 \\
2 & 72.69 & 72.04 \\
3 & 73.18 & 72.50 \\
4 & NA & 72.50 \\
5 & NA & 72.46 \\
6 & NA & 72.53 \\
\hline
\end{tabular}

The slight decrease in weights on day 2 was associated with a temporary instability in the humidity control system; no analysis was carried out on that day. Weights could no longer be taken from multiple boxes once machine smoking began, but one box (26) was set aside from the main analysis of smoke yields and its weight monitored until the final day of smoking. $\mathrm{NA}=$ not applicable

Table 5 Summary statistics for number of puffs and smoke yields (mg per cigarette) for the 26 recruited $R Y O$ smokers

\begin{tabular}{lllll}
\hline & Puffs & CO & Nicotine & Tar \\
\hline Mean & 12.3 & 10.9 & 1.3 & 15.7 \\
Range & $9.4-15.7$ & $5.5-14.1$ & $0.9-1.8$ & $9.9-21.0$ \\
Sample standard deviation & 1.5 & 2.2 & 0.2 & 2.8 \\
$F / F_{\text {crit }}$ & 5.1 & 2.2 & 2.3 & 2.1 \\
& significant & significant & significant & significant \\
\hline
\end{tabular}

The tabulated values are calculated from the mean values recorded for each of the 26 recruits. A one-way analysis of variance was performed on the raw data: $F / F_{\text {crit }}$ is the ratio of the calculated $F$ ratio value to the critical $F$ ratio for $99 \%$ confidence.
ond

$\mathrm{CO}=$ carbon monoxide. contained 0.919 g. Most participants tended to use a similar weight of tobacco for each of their cigarettes: on average, the standard deviation of tobacco weights for the 20 cigarettes rolled by each participant was $0.058 \mathrm{~g}$ (the average coefficient of variation was $11.7 \%$ ).

An indication of the distribution of diameters for these cigarettes was obtained from the choice of labyrinth seal used to attach each cigarette to the smoking machine. The seals were chosen to match the cigarette diameters as closely as possible but without distortion of the butt ends. For $85 \%$ of the cigarettes, a $6 \mathrm{~mm}$ seal was selected; $13 \%$ required a $5 \mathrm{~mm}$ seal, and $2 \%$ a $7 \mathrm{~mm}$ seal.

SMOKE YIELDS FROM CIGARETTES MADE BY 26 RECRUITED RYO SMOKERS

Machine smoking over an interval of several days was required to process the cigarettes prepared by the recruits. This unavoidable interval necessitated the use of storage conditions that would preserve the initial moisture content of tobacco when it was rolled. The gross weights of 15 of the boxes, each containing all the cigarettes rolled by one survey participant, were monitored from collection until the start of machine smoking while stored under the constant environmental conditions. Additionally one box was left intact to monitor the conditions during the intervening period until the last smoking run (table 4). There was no marked trend in the variation of the weights of boxes with time, and this gives a strong indication that moisture content varied negligibly with time under the chosen storage conditions.

Summary statistics for number of puffs, and for yields of $\mathrm{CO}$, nicotine, and tar, are presented in table 5. The sampling structure for the commissioned survey was that each of the 26 recruits would produce 20 cigarettes, and that each such batch of 20 would be smoked as five samples of four cigarettes. The analytical data returned for each smoker thus allowed within-smoker and between-smoker variability to be examined by one-way analysis of variance. Variance ratio testing indicated that there was a significant difference both between and within smokers for all three smoke yields. The variance ratio calculated for puff number was noticeably higher than those from the smoke yields (table 5). Error bars on the figures indicate the extent of individual standard deviations. tobacco was reduced to $0.59 \mathrm{~g}$ so that the same weight per unit length was used as for plain cigarettes containing $0.75 \mathrm{~g}$ of tobacco. The slimline product was substantially less effective in reducing smoke yields. This finding was supported by the lower nicotine retention of this filter (table 3).

SURVEY OF 26 RECRUITED RYO SMOKERS: PHYSICAL CHARACTERISTICS OF THE CIGARETTES PRODUCED

The overall average weight of tobacco used per cigarette by the 26 participants was $0.505 \mathrm{~g}$. The average weights of tobacco used per cigarette by individual participants ranged from $0.328 \mathrm{~g}$ to $0.818 \mathrm{~g}$. The lightest cigarette contained $0.226 \mathrm{~g}$ of tobacco whereas the heaviest
ASSOCIATION BETWEEN TOBACCO WEIGHT AND SMOKE YIELDS smoke yields was studied in the preliminary study of cigarettes prepared under laboratory conditions, and in the consumer survey.

As expected, cigarettes made in the laboratory with $0.75 \mathrm{~g}$ of tobacco produced, on average, substantially higher smoke yields than those made with $0.50 \mathrm{~g}$. This applied regardless of the cigarette paper chosen for the comparison (table 2). However, in general, the yields did not decrease in proportion to the weight of tobacco, particularly in the case of the more porous paper. These findings must be
The association between tobacco weight and 

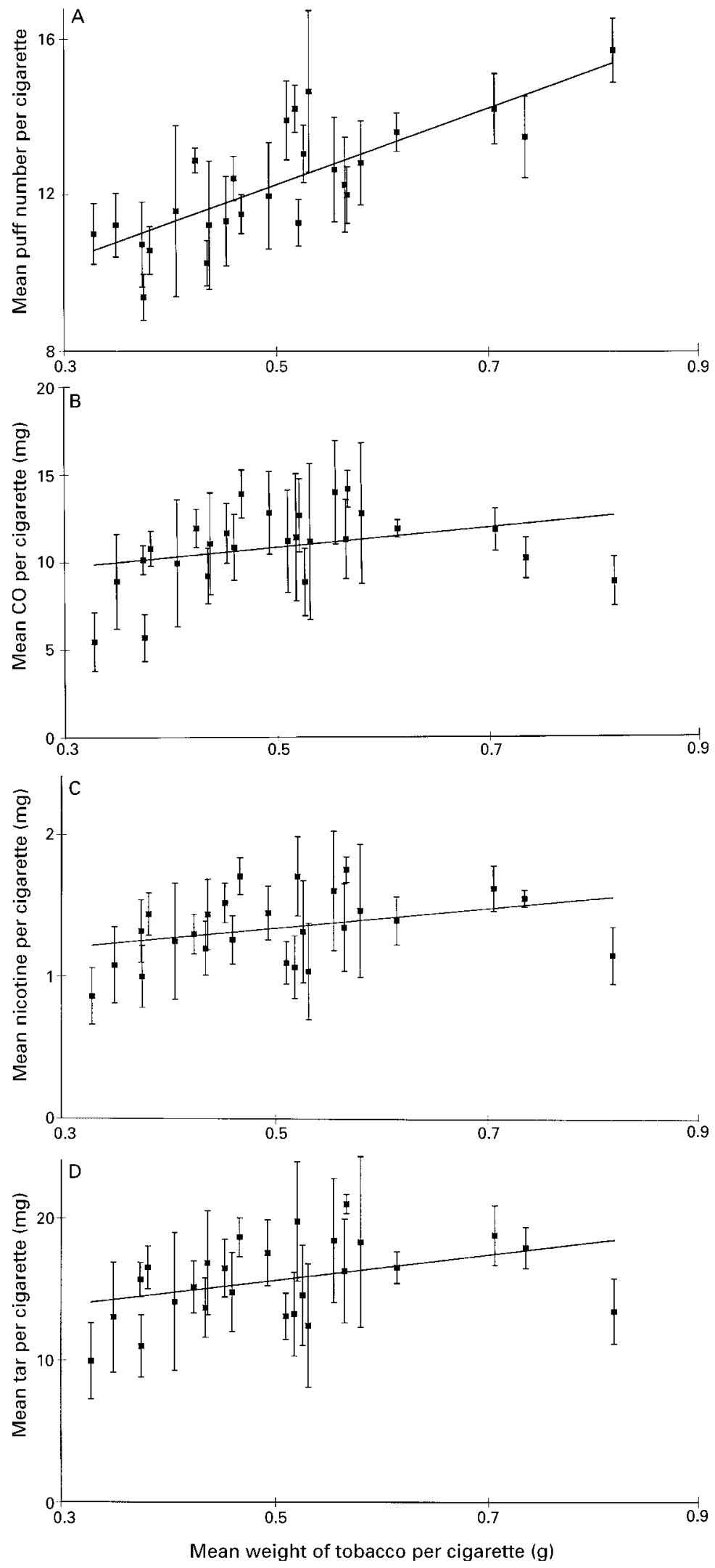

Figure 1 Association between tobacco weight and number of puffs and tobacco weight and smoke yields for the 26 recruited roll-your-own smokers. Each point represents the mean of the plotted variable for one recruit (error bars represent \pm 1 sample standard deviation), calculated from determinations made using sets of four cigarettes taken randomly from those rolled by each person. (A) Puffs per cigarette; (B) carbon monoxide yield; (C) nicotine yield; (D) tar yield. The trend lines were derived by least squares linear regression. interpreted cautiously because of the difference in rolling technique that was necessary for the lower weight of tobacco; however, it may be surmised that in using a smaller amount of tobacco the cigarette diameter is likely to be smaller. To contain the tobacco securely, the paper may need to be wrapped around on itself. Such an action will modify its porosity downwards.

The data from the consumer survey, as shown in figure 1A-D, shows the observed association between the mean weight of tobacco per cigarette and each of the four analytical parameters measured (number of puffs, CO, nicotine, and tar). The strongest association was observed between the weight of tobacco and the number of puffs (an expression of the smoking time) (as shown in figure $1 \mathrm{~A}$, for which the Pearson product moment correlation coefficient $(r)$ was 0.78 . A slight upward trend in yield with increasing weight was also observed for the other analyses (figures 1B-D), although there is significant scatter of individual smoker results about the regression lines. In particular, the yields for the smoker who produced cigarettes having the highest average weight were lower than might have been predicted; it was noticed that these cigarettes were loosely packed.

The one smoker who used a rolling machine during the survey produced cigarettes with the highest yields for CO, nicotine, and tar. The average weight of tobacco used, and the correspondence of this with smoke yields, were unexceptional for the one female participant. The weights of tobacco, number of puffs, and smoke yields obtained by smoking cigarettes from the two recruits who stated that they normally incorporated a filter in their cigarettes fell within the range of yields of the remaining recruits and were therefore not excluded from the final result summary.

\section{Discussion}

Cigarettes made and smoked under standardised conditions in the laboratory showed substantial differences in smoke yields, as observed between, on the one hand, two brands of cigarette papers of similar (and lower) porosity and, on the other, a highly porous brand. It should not, however, be assumed from this study that the porosity of cigarette paper is the only property of the paper that has an effect on smoke yields. The chemical composition of the paper is likely to play a part, although a systematic study is difficult without reliance on detailed information about paper manufacture from the tobacco industry. A comparison of the porosities of 13 brands of papers, taking into consideration market share data, showed (results not shown) that most papers sold in the United Kingdom are of similar porosity to the lower porosity varieties studied. A Dutch report ${ }^{14}$ indicates that papers used in the Netherlands are frequently of a similar porosity to the higher porosity brand used in this study. Cigarette tubes are an alternative to cigarette papers for RYO consumers in some countries, but are not generally available in the United Kingdom. ${ }^{3}$ 
This study reinforces the perception that inclusion of filters in RYO cigarettes can reduce the yields of tar and nicotine to the smoker, and indicates that the choice of filter product has an important effect on smoke yields. The use of filters in combination with hand-rolled cigarettes is not common in United Kingdom. ${ }^{3}$ There are grounds to suppose that classification of cigarette papers and filters according to their respective influences on smoke yields might be useful.

Although there are differences of detail between the methods used, the smoke yields presented here for cigarettes made reproducibly in the laboratory are comparable with those previously reported from the Netherlands ${ }^{14}$ and from Norway. ${ }^{15}$ The Dutch study has shown that a butt length of $27 \mathrm{~mm}$ reduces yields by approximately $15 \%$ from those obtained with the $23 \mathrm{~mm}$ butt length chosen for our present project. The much higher cost of RYO tobacco in the United Kingdom is likely to influence many smokers to smoke to a minimum butt length. Therefore we did not consider it necessary in a British study to employ a butt length greater than the standard $23 \mathrm{~mm}$ for plain manufactured cigarettes or those with a filter length of $15 \mathrm{~mm}$.

The weights of tobacco used in this study, where these were determined by the investigators, were chosen from experimental considerations and on the basis of current British practice. A draft standard method for the determination of tar and nicotine yields of RYO cigarettes has been proposed in Germany $^{26}$ using $1 \mathrm{~g}$ of tobacco, and this weight has also been selected for work in The Netherlands ${ }^{14}$ where the cost of hand-rolling tobacco is much lower than in the United Kingdom. The average weight of tobacco used per cigarette by the participants of this study was $0.505 \mathrm{~g}$. This agrees well with a reported figure of $0.49 \mathrm{~g},{ }^{3}$ although the same paper cites more recent unpublished data which indicates a somewhat lower weight. The limited variation observed in weight per cigarette for a given consumer may indicate that most RYO consumers are experienced in the consistent production of cigarettes having physical characteristics suited to their tastes. Consequently, information on smoke yields classified according to physical characteristics of RYO cigarettes may be of direct use to the consumer.

Plain cigarettes containing the same tobacco and the same paper (B) were prepared and smoked both under laboratory conditions (table 2) and as part of the survey of recruited RYO smokers (table 5). Yields from cigarettes made by the 26 members of the public were in general much lower than from cigarettes made under laboratory conditions. However, more than half of the mean tar yields of the cigarettes made by the public were above $15 \mathrm{mg}$ per cigarette (the 1991 regulatory limit for manufactured cigarettes). Nicotine yields were found in general to be higher than those obtained from manufactured cigarettes. Currently the Laboratory of the Government
Chemist estimates that approximately $8 \%$ of manufactured brands on sale in the United Kingdom have a declared nicotine yield greater than $1.1 \mathrm{mg}$ per cigarette, whereas $77 \%$ of the RYO consumers included in our survey produced cigarettes giving average yields above this figure.

The results of the consumer survey demonstrated highly significant betweenconsumer variation in the three smoke yields, which could not be explained by inconsistent smoking behaviour within-consumer or by variations in cigarette handling and analysis procedures. The between-consumer variation in number of puffs was even more noticeable. It is not possible from the analysis of this data to ascertain whether puff number and smoke yields are influenced by variations in an underlying causal parameter. A noticeable proportion of these cigarettes, in contrast to laboratory-manufactured cigarettes, extinguished on occasions during the interval between puffs taken by the smoking machine. This may reflect a habitual intention on the part of some RYO consumers not to smoke a whole cigarette in one session. Alternatively the consumer puffing rate could be higher than that for machine smoking.

We found a positive correlation between the average weight of tobacco used by the $26 \mathrm{RYO}$ consumers and each of the corresponding average smoke yields-figures $1 \mathrm{~B}, \mathrm{C}$, and $\mathrm{D}$-but the correlation was poor in each case. Relatively low smoke yields, especially for carbon monoxide, were obtained for average tobacco weights at the upper end of the recorded range. Because the materials available for the preparation of all these cigarettes were kept constant, this suggests that the manner of rolling and the physical characteristics of the cigarettes have an important effect on yields. The survey points to the possibility that RYO smokers who employ a rolling machine might be at considerably higher risk than manual rollers, although this hypothesis is founded on the high smoke yields from cigarettes produced by the one and only machine roller included, and requires further substantiation.

It is important to keep in mind the complementary roles of the two phases of this investigation. A previous study ${ }^{27}$ conducted in Canada has suggested that for RYO products manufactured from similar types of tobacco, the brand used has little or no influence on the smoke yields obtained under laboratory conditions. Consequently, we have not sought to address the issue of whether one brand of hand-rolling tobacco is more deleterious to health than another, but have used a single popular tobacco brand throughout the reported work. In an attempt to describe the association between RYO smoking and health, smoke yields have been determined on RYO cigarettes prepared under carefully specified conditions, and the effects of changes in three key parameters - types of paper, addition of a filter, and the weight of tobacco-have been observed. The consumer survey work has indicated that if the first parameter (paper) is kept constant and the third (weight of tobacco) is 
allowed to vary, the resulting smoke yields do not correlate strongly with the variable parameter. If one accepts this study as approximately representative of the RYO consumer population (given the practical constraints on the sample of consumers obtained), there are clearly further consumer-determined physical parameters that influence the smoke yields of RYO cigarettes. Chief among these are the diameter and the longitudinal packing profile of the cigarettes rolled. Further research could usefully concentrate on these crucial parameters so that regulatory authorities may be in a better position to provide advice and education, and to institute control measures, relating to the smoking of RYO cigarettes.

Notwithstanding such unknowns there is evidence showing that, on average, tar yields of hand-rolled cigarettes are higher than most manufactured brands currently on sale in the United Kingdom. It should also be of concern that both this study and others ${ }^{14}{ }^{15} 28$ indicate that nicotine yields from hand-rolled cigarettes significantly extend the range encountered from manufactured brands. As a possible consequence it has been reported ${ }^{28}$ that nicotine dependence among hand-rolling smokers is greater than that among those smoking manufactured cigarettes.

The variability of individual consumers' smoking behaviour must not be forgotten. It has been reported widely that smoke yields derived by standard smoking machine methodology cannot reflect the true exposure of all smokers to the hazardous components of tobacco smoke. In the case of manufactured cigarettes, it has been shown ${ }^{29-31}$ that smokers will compensate, or change their smoking pattern, to enable them to receive a measured amount of nicotine, the driving force behind the urge to smoke. It is likely that a similar type of behaviour will be found in smokers of handrolled cigarettes, but such information can only be ascertained by monitoring actual ingestion levels through, for example, measurement of nicotine and cotinine levels in body fluids. A comparison of the hazards of RYO products and other tobacco goods would be facilitated if physiological monitoring could be carried out as function of the physical parameters of the cigarettes smoked.

This work was fully funded by the United Kingdom Department of Health Smoking Policy Unit, Wellington House, Waterloo Road, London SE1 8UG, United Kingdom. The authors acknowledge the assistance given by Dr John Francis in the preparation of this paper.

1 Anon. Rolling tobacco-an unbalanced situation. Tobacco 1995; May/Jun:10-12.

2 Cole J. Hand-rolling havens. World Tobacco 1996;153:56-60.

3 Dymond HF. A survey of roll-your-own tobacco use and practice and considerations for the analysis of smoking articles made from it. Tobacco Sci 1996;40:82-6.
4 Bennet N, Jarvis L, Rowlands O, et al. Living in Britain: results from the 1994 General Household Survey. London: results from the

5 Doll R, Hill AB. Smoking and carcinoma of the lung. BMF 1976;ii: $739-48$.

6 Doll R, Peto R, Wheatley K, et al. Mortality in relation to smoking: 40 years' observations on male British doctors. BMF 1994;309:901-11.

7 Royal College of Physicians. Health or smoking? London: Pitman, 1983.

8 Engeland A, Haldorsen T, Andersen A, et al. The impact of smoking habits on lung cancer risk. I: 28 years' observation of 26,000 Norwegian men and women. Cancer Causes Control 1996;7:366-76.

9 Tuyns AJ, Esteve J. Pipe, commercial and hand-rolled cigarette smoking in oesophageal cancer. Int 7 Epidemiol 1983 12:110-13.

10 De Stefani E, Barrios E, Fierro L. Black (air-cured) and blond (flue-cured) tobacco and cancer risk III: oesophageal cancer. Eur 7 Cancer 1993;29A:763-6.

11 De Stefani E, Oreggia F, Rivero S, et al. Hand-rolled cigarette smoking and risk of cancer of the mouth, pharynx and larynx. Cancer 1992;70:679-82.

12 Kune GA, Kune S, Vitetta L, et al. Smoking and colorectal cancer risk: data from the Melbourne colorectal cancer study and brief review of literature. Int $\mathcal{f}$ Cancer 1992;50:369-72.

13 Independent Scientific Committee on Smoking and Health. Fourth report. London: HMSO, 1988.

14 de Kok A, Besamusca EW, Vreeker CP, et al. Roll-your-own: a delicate subject. A study of the tar and nicotine content of the 42 roll your own brands sold in the Netherlands. Report $A L$ TAB-1992-1. Alkmaar, Netherlands: Inspectie Gezondheidsbescherming, 1993.

15 Hauknes A. Analysis of hazardous substances in rolling tobaccos. Oslo, Norway: National Council on Tobacco and Health, 1994

16 Rickert WS, Robinson JC, Bray DF, et al. Characterization of tobacco products: a comparative study of the tar, nicotine, and carbon monoxide yields of cigars, manufactured cigarettes, and cigarettes made from fine-cut tobacco. Prev Med 1985;14:226-33.

17 Mitacek EJ, Brunnemann KD, Polednak AP, et al. Composition of popular tobacco products in Thailand, and its relevance to disease prevention. Prev Med 1991;20:764-73.

18 Dymond H. Making habits of roll-your-own smokers in the Netherlands and tar and nicotine yields from the resultant products. Tobacco Sci 1996;40:87-91.

19 Darrall KG. Smoking machine parameters and cigarette smoke yields. Sci Total Env 1988;74:263-78

20 Routine analytical cigarette smoking machine: Part 1. Specification and standard conditions. ISO 3308. Geneva, Switzerland: International Standards Organisation, 1991.

21 Determination of total and nicotine free dry particulate matter using a routine analytical smoking machine. ISO 4387. Geneva, Switzerland: International Standards Organisation, 1991

22 Determination of nicotine in smoke condensate of cigarettes (gas chromatographic method). ISO 10315. Geneva, Switzerland: International Standards Organisation, 1991.

23 Determination of water in smoke condensate of cigarette (gas chromatographic method). ISO 10362-1. Geneva, Switzerland: International Standards Organisation, 1991.

24 Determination of carbon monoxide in vapour phase of cigarette smoke (NDIR method). ISO 8454 , Geneva, Switzerland: International Standards Organisation, 1995.

25 Atmosphere for conditioning and testing fine-cut tobacco and fine-cut smoking articles. Coresta Recommended Method No.42. Coresta Bull 1997;1:39-43.

26 Deutsches Institute für Normung (DIN). DIN 10 256. Bestimmung der Ausbeutefahigkeit von Feinscnitt-Tabak. Berlin, Germany: DIN, 1989

27 Kaiserman MJ, Rickert WS. Handmade cigarettes: it's the tube that counts. Am F Public Health 1992;82:107-9.

28 Kraft P, Svendsen T, Haukes A. Nicotine dependence among Norwegian smokers and possible consequences for preventative actions. Tidsskr Nor Laegeforen 1997;117:340-

29 Herning RI, Jones RT, Bahman J, et al. Puff volume increases when low nicotine cigarettes are smoked. $B M \mathcal{F}$ 1981;283:187-9.

30 Benowitz NL, Hall SH, Herning RI, et al. Smokers of low yield cigarettes do not consume less nicotine. New England f Med 1983;309:139-42.

31 Djordjevic MV, Fan J, Ferguson S, et al. Self-regulation of smoking intensity. I. Smoke yields of low-nicotine, low-tar cigarettes. Carcinogenesis 1995;16:2015-21. 